\title{
On vertex-stabilizers of bipartite dual polar graphs
}

\author{
Štefko Miklavič \\ Faculty of Mathematics, Natural Sciences and Information Technologies \\ University of Primorska, SI-6000 Koper, Slovenia
}

Received 18 September 2009, accepted 15 December 2009, published online 3 February 2010

\begin{abstract}
Let $X, Y$ denote vertices of a bipartite dual polar graph, and let $G_{X}$ and $G_{Y}$ denote the stabilizers of $X$ and $Y$ in the full automorphism group of this graph. In this paper, a description of the orbits of $G_{X} \cap G_{Y}$ in the cases when the distance between $X$ and $Y$ is 1 or 2 , is given.
\end{abstract}

Keywords: Dual polar graphs, automorphism group, quadratic form, isotropic subspace.

Math. Subj. Class.: 05E18, 05E30

\section{Preliminaries and introductory remarks}

Let $q$ denote a prime power, let $G F(q)$ denote a finite field with $q$ elements, and let $d$ denote a positive integer. Let $V=G F(q)^{2 d}$ denote the vector space over $G F(q)$ of dimension $2 d$, consisting of column vectors with entries in $G F(q)$. We define a map $Q: V \rightarrow G F(q)$ as follows. For $u=\left(u_{1}, u_{2}, \ldots, u_{2 d}\right)^{t} \in V$ we let

$$
Q(u)=\sum_{i=1}^{d} u_{2 i-1} u_{2 i}
$$

The form $Q$ is a quadratic form on $V$, that is, $Q(\lambda u)=\lambda^{2} Q(u)(\lambda \in G F(q), u \in V)$, and

$$
f(u, v)=Q(u+v)-Q(u)-Q(v) \quad(u, v \in V)
$$

is a symmetric bilinear form on $V$. The form $Q$ is usually called hyperbolic quadric. Note that for vectors $u=\left(u_{1}, u_{2}, \ldots, u_{2 d}\right)^{t}$ and $v=\left(v_{1}, v_{2}, \ldots, v_{2 d}\right)^{t}$ of $V$ we have

$$
f(u, v)=\sum_{i=1}^{d}\left(u_{2 i-1} v_{2 i}+u_{2 i} v_{2 i-1}\right) .
$$

E-mail address: stefko.miklavic@upr.si (Štefko Miklavič) 
A vector $v \in V$ is called isotropic, if $Q(v)=0$. A subspace $U$ of $V$ is called isotropic, if $Q(u)=0$ for every $u \in U$, and it is called maximal isotropic, if it is maximal (with respect to inclusion) in the set of all isotropic subspaces of $V$. It turns out that the dimension of every maximal isotropic subspace is $d$ (see, for example, [1, Theorem 3.10] or [10, Lemma 3]). Observe that if $u, v \in V$ belong to the same isotropic subspace of $V$, than $Q(\lambda u+\mu v)=0$ for every $\lambda, \mu \in G F(q)$. Furthermore,

$$
f(u, v)=Q(u+v)-Q(u)-Q(v)=0 .
$$

Conversely, if $u$ and $v$ are isotropic with $f(u, v)=0$, then $\langle u, v\rangle$ is an isotropic subspace of $V$. Indeed, for $\lambda, \mu \in G F(q)$ we have

$$
Q(\lambda u+\mu v)=\lambda^{2} Q(u)+\mu^{2} Q(v)+\lambda \mu f(u, v)=0 .
$$

We now define the dual polar graph $D_{d}(q)$ on $V$. The vertex-set $V\left(D_{d}(q)\right)$ of $D_{d}(q)$ is the set of all maximal isotropic subspaces of $V$. Vertices $X, Y \in V\left(D_{d}(q)\right)$ are adjacent in $D_{d}(q)$ if and only if the dimension of $X \cap Y$ is $d-1$. Let $\partial$ denote the path-length distance function on $D_{d}(q)$. It is easy to see that $\partial(X, Y)=i$ if and only if $\operatorname{dim}(X \cap Y)=d-i$ $\left(X, Y \in V\left(D_{d}(q)\right)\right)$. The following facts about $D_{d}(q)$ can be found, for example, in [2, Section 9.4]. The graph $D_{d}(q)$ is bipartite with diameter $d$ and with $\prod_{i=0}^{d-1}\left(q^{d-i-1}+1\right)$ vertices. For convenience let

$$
b_{i}=q^{i} \frac{q^{d-i}-1}{q-1}, \quad c_{i}=\frac{q^{i}-1}{q-1} \quad \text { and } \quad k_{i}=\frac{b_{0} b_{1} \cdots b_{i-1}}{c_{1} c_{2} \cdots c_{i}}
$$

for $0 \leq i \leq d$. The graph $D_{d}(q)$ is regular with valency $b_{0}=k_{1}$. For $X \in V\left(D_{d}(q)\right)$ and an integer $0 \leq i \leq d$ we set $S_{i}(X)=\left\{Z \in V\left(D_{d}(q)\right) \mid \partial(X, Z)=i\right\}$.

Let $G L(V)$ denote the general linear group of $V$. Then $\sigma \in G L(V)$ is called isometry of $V$, if $Q(\sigma(v))=Q(v)$ for every $v \in V$. It follows from (1.2) that if $\sigma$ is an isometry of $V$, then $f(u, v)=f(\sigma(u), \sigma(v))$ for $u, v \in V$. The group of all isometries of $V$ is called the orthogonal group for $Q$, and is denoted by $O_{2 d}^{+}(q)$. Note that every $\sigma \in O_{2 d}^{+}(q)$ acts on $V\left(D_{d}(q)\right)$ as an automorphism of $D_{d}(q)$. The full automorphism group $G$ of $D_{d}(q)$ acts distance-transitively on $V\left(D_{d}(q)\right)$, that is, for $X, Y, Z, W \in V\left(D_{d}(q)\right)$ with $\partial(X, Y)=$ $\partial(Z, W)$ there exists $\sigma \in G$ such that $\sigma(X)=Z$ and $\sigma(Y)=W$ (see, for example, [2, Table 6.1]). Recall that every distance-transitive graph is also distance-regular in the sense of [2, Section 4.1].

Pick $X, Y \in V\left(D_{d}(q)\right)$ and let $G_{X}$ and $G_{Y}$ denote the stabilizers of $X$ and $Y$ in $G$, respectively. Since $G$ acts distance-transitively on $V\left(D_{d}(q)\right)$, the orbits of $G_{X}$ are precisely the sets $S_{i}(X)(0 \leq i \leq d)$. In this paper we examine the orbits of $G_{X} \cap G_{Y}$. These orbits play an important role in the theory of Terwilliger algebras of $D_{d}(q)$. This role is especially important in the case when $\partial(X, Y) \in\{1,2\}$, see [6]. For the definition and more background on Terwilliger algebras of distance-regular graphs see [3, 4, 7, 8, 9].

In this paper we give a description of the orbits of $G_{X} \cap G_{Y}$ when $\partial(X, Y) \in\{1,2\}$. To do this, we consider the following situation for the rest of this paper.

Notation 1.1. Let $q$ denote a prime power, let $G F(q)$ denote a finite field with $q$ elements, and let $d$ denote a positive integer. Let $V=G F(q)^{2 d}$ denote the vector space over $G F(q)$ of dimension $2 d$, consisting of column vectors with entries in $G F(q)$. Let $Q$ and $f$ be as defined in (1.1) and (1.2). Let $D_{d}(q)$ denotes the bipartite dual polar graph over $V$, 
and let $b_{i}, c_{i}$ and $k_{i}$ be as in (1.6). Fix $X, Y \in V\left(D_{d}(q)\right)$. For $0 \leq i, j \leq d$ let $D_{j}^{i}=$ $D_{j}^{i}(X, Y)=S_{i}(X) \cap S_{j}(Y)$. Let $G_{X}$ and $G_{Y}$ denote the stabilizers of $X$ and $Y$ in the full automorphism group $G$ of $D_{d}(q)$.

Our paper is organised as follows. In Section 2 we state some results about maximal isotropic subspaces that we need later. In Section 3 (Section 4, respectively) we describe the orbits of $G_{X} \cap G_{Y}$ in the case when $\partial(X, Y)=1(\partial(X, Y)=2$, respectively). In what follows we use the same symbols (capital letters) for the vertices of $D_{d}(q)$ and for the maximal isotropic subspaces of $V$; this should cause no confusion.

\section{Maximal isotropic subspaces}

In this section we state some results about maximal isotropic subspaces of $V$ that we need later. The first one is known as Witt's lemma (see, for example, [1, Theorem 3.9]).

Lemma 2.1. With reference to Notation 1.1, let $U$ and $W$ be subspaces of $V$, and let $\sigma_{U}: U \rightarrow W$ be a bijective linear map satisfying $Q\left(\sigma_{U}(u)\right)=Q(u)$ for every $u \in U$. Then there is an isometry of $V$ which extends $\sigma_{U}$.

Lemma 2.2. With reference to Notation 1.1, let $U$ and $W$ be maximal isotropic subspaces of $V$ with $\operatorname{dim}(U \cap W)=d-i$ for some $1 \leq i \leq d$. Pick linearly independent vectors $u_{1}, \ldots, u_{i} \in U \backslash W$ and linearly independent vectors $w_{1}, \ldots, w_{i} \in W \backslash U$. Let $F$ be the $i \times i$ matrix with $(j, \ell)$-entry equal to $f\left(u_{j}, w_{\ell}\right)$. Then the determinant of $F$ is nonzero.

Proof. First note that $F$ is a nonzero matrix. Namely, if $f\left(u_{j}, w_{\ell}\right)=0$ for every $1 \leq$ $j, \ell \leq i$, then a subspace generated by $U$ and $W$ is isotropic subspace of dimension $d+i$, a contradiction. Suppose now that $\operatorname{det}(F)=0$. Then the columns of $F$ are linearly dependent vectors of $G F(q)^{i}$, that is, there exist scalars $\lambda_{j}(1 \leq j \leq i)$ which are not all equal to zero, such that for each $1 \leq \ell \leq i$ we have

$0=\lambda_{1} f\left(u_{\ell}, w_{1}\right)+\lambda_{2} f\left(u_{\ell}, w_{2}\right)+\cdots+\lambda_{i} f\left(u_{\ell}, w_{i}\right)=f\left(u_{\ell}, \lambda_{1} w_{1}+\lambda_{2} w_{2}+\cdots+\lambda_{i} w_{i}\right)$.

Note that $w=\lambda_{1} w_{1}+\lambda_{2} w_{2}+\cdots+\lambda_{i} w_{i}$ is nonzero, since $w_{1}, w_{2}, \ldots, w_{i}$ are linearly independent. Multiplying the above equation with an arbitrary scalar $\mu_{\ell}$ gives us $\mu_{\ell} f\left(u_{\ell}, w\right)=0$. Adding the obtained equations we get

$$
\sum_{\ell=1}^{i} \mu_{\ell} f\left(u_{\ell}, w\right)=f\left(\mu_{1} u_{1}+\mu_{2} u_{2}+\cdots+\mu_{i} u_{i}, w\right)=0 .
$$

This implies that $f(u, w)=0$ for every $u \in U$. By (1.5), the subspace generated by $U$ and $w$ is isotropic with dimension $d+1$, a contradiction. Therefore, $\operatorname{det}(F) \neq 0$.

Lemma 2.3. With reference to Notation 1.1, let $U, U_{1}, W$ and $W_{1}$ be maximal isotropic subspaces of $V$ with $\operatorname{dim}(U \cap W)=\operatorname{dim}\left(U_{1} \cap W_{1}\right)=d-i$ for some $1 \leq i \leq d$. Let $u_{1}, u_{2}, \ldots, u_{d}$ be a basis of $U$ such that $u_{i+1}, \ldots, u_{d}$ is a basis of $U \cap W$. Let $w_{1}, \ldots, w_{i} \in$ $W$ be such that $w_{1}, \ldots, w_{i}, u_{i+1}, \ldots, u_{d}$ is a basis of $W$. Let $v_{1}, v_{2}, \ldots, v_{d}$ be a basis of $U_{1}$ such that $v_{i+1}, \ldots, v_{d}$ is a basis of $U_{1} \cap W_{1}$. Let $z_{1}, \ldots, z_{i} \in W_{1}$ be such that $z_{1}, \ldots, z_{i}, v_{i+1}, \ldots, v_{d}$ is a basis of $W_{1}$. Then there exists an isometry $\sigma$ of $V$, such that $\sigma\left(u_{j}\right)=v_{j}(1 \leq j \leq d)$ and $\sigma\left(w_{j}\right) \in\left\langle z_{1}, \ldots, z_{i}\right\rangle(1 \leq j \leq i)$. 
Proof. We first define a bijective linear map $\bar{\sigma}$ from a subspace generated by $U$ and $W$ to a subspace generated by $U_{1}$ and $W_{1}$, such that $\bar{\sigma}\left(u_{j}\right)=v_{j}(1 \leq j \leq d)$ and $\bar{\sigma}\left(w_{j}\right) \in$ $\left\langle z_{1}, \ldots, z_{i}\right\rangle(1 \leq j \leq i)$. We will then show that $\bar{\sigma}$ extends to an isometry of $V$. We now define $\bar{\sigma}\left(w_{j}\right)(1 \leq j \leq i)$. Let $F$ denote an $i \times i$ matrix with $(j, \ell)$-entry equal to $f\left(v_{j}, z_{\ell}\right)$. For $1 \leq \ell \leq i$ consider the following system of linear equations in variables $\alpha_{1}^{\ell}, \alpha_{2}^{\ell}, \ldots, \alpha_{i}^{\ell}$ :

$$
F\left(\alpha_{1}^{\ell}, \alpha_{2}^{\ell}, \ldots, \alpha_{i}^{\ell}\right)^{t}=\left(f\left(u_{1}, w_{\ell}\right), f\left(u_{2}, w_{\ell}\right), \ldots, f\left(u_{i}, w_{\ell}\right)\right)^{t} .
$$

Note that this system has a unique solution since $F$ is nonsingular by Lemma 2.2. For convenience, we denote the solutions of this system also by $\alpha_{1}^{\ell}, \alpha_{2}^{\ell}, \ldots, \alpha_{i}^{\ell}$. For $1 \leq \ell \leq i$ we let

$$
\bar{\sigma}\left(w_{\ell}\right)=\alpha_{1}^{\ell} z_{1}+\alpha_{2}^{\ell} z_{2}+\cdots+\alpha_{i}^{\ell} z_{i}
$$

We extend $\bar{\sigma}$ to a linear map from $\langle U, W\rangle$ to $\left\langle U_{1}, W_{1}\right\rangle$ in a natural way:

$$
\begin{aligned}
& \bar{\sigma}\left(\lambda_{1} u_{1}+\cdots+\lambda_{d} u_{d}+\mu_{1} w_{1}+\cdots+\mu_{i} w_{i}\right)= \\
& \lambda_{1} \bar{\sigma}\left(u_{1}\right)+\cdots+\lambda_{d} \bar{\sigma}\left(u_{d}\right)+\mu_{1} \bar{\sigma}\left(w_{1}\right)+\cdots+\mu_{i} \bar{\sigma}\left(w_{i}\right)
\end{aligned}
$$

for $\lambda_{1}, \ldots, \lambda_{d}, \mu_{1}, \ldots, \mu_{i} \in G F(q)$.

We now show that $\bar{\sigma}$ is a bijection. To do this, it is enough to show that $\bar{\sigma}\left(w_{\ell}\right)(1 \leq \ell \leq$ $i$ ) are linearly independent. Let $A$ be an $i \times i$ matrix with $(j, \ell)$-entry equal to $\alpha_{j}^{\ell}$. Observe that $\bar{\sigma}\left(w_{\ell}\right)(1 \leq \ell \leq i)$ are linearly independent if and only if $A$ is nonsingular. Let $F_{1}$ denote an $i \times i$ matrix with $(j, \ell)$-entry equal to $f\left(u_{j}, w_{\ell}\right)$. The matrix $F_{1}$ is nonsingular by Lemma 2.2. Furthermore, it follows from (2.1) that $F \cdot A=F_{1}$, implying that $A$ is nonsingular.

We now show that $\bar{\sigma}$ preserves $Q$. Pick arbitrary $v \in\langle U, W\rangle$ :

$$
v=\sum_{j=1}^{d} \alpha_{j} u_{j}+\sum_{j=1}^{i} \beta_{j} w_{j}
$$

By (1.2) and (1.4),

$$
Q(v)=\sum_{r=1}^{i} \sum_{s=1}^{i} \alpha_{r} \beta_{s} f\left(u_{r}, w_{s}\right) .
$$

Let us now compute $Q(\bar{\sigma}(v))$. By (1.2) and (1.4) we first get

$$
Q(\bar{\sigma}(v))=\sum_{r=1}^{i} \sum_{s=1}^{i} \alpha_{r} \beta_{s} f\left(\bar{\sigma}\left(u_{r}\right), \bar{\sigma}\left(w_{s}\right)\right) .
$$

By (2.2) and since $\sigma\left(u_{r}\right)=v_{r}$ we further find

$$
f\left(\bar{\sigma}\left(u_{r}\right), \bar{\sigma}\left(w_{s}\right)\right)=f\left(v_{r}, \alpha_{1}^{s} z_{1}+\cdots+\alpha_{i}^{s} z_{i}\right)=\alpha_{1}^{s} f\left(v_{r}, z_{1}\right)+\cdots+\alpha_{i}^{s} f\left(v_{r}, z_{i}\right) .
$$

Finally, by (2.1), the above expression is equal to $f\left(u_{r}, w_{s}\right)$. Therefore, $Q(v)=Q(\bar{\sigma}(v))$. By Lemma 2.1 there exists an isometry $\sigma$ of $V$ which extends $\bar{\sigma}$. This completes the proof. 
Lemma 2.4. With reference to Notation 1.1, let $U$ be a $(d-1)$-dimensional isotropic subspace of $V$. Then $U$ is contained in exactly two maximal isotropic subspaces of $V$.

Proof. By [2, p. 274], the number of isotropic $k$-dimensional subspaces of $V$ containing a given isotropic $(k-1)$-dimensional subspace of $V$ is $\left(q^{d-k+1}-1\right)\left(q^{d-k}+1\right) /(q-1)$. The result follows.

\section{The case $\partial(X, Y)=1$}

With reference to Notation 1.1, in this section we describe the orbits of $G_{X} \cap G_{Y}$ when $\partial(X, Y)=1$. We first determine the size of the $D_{j}^{i}(0 \leq i, j \leq d)$.

Lemma 3.1. With reference to Notation 1.1 assume that $\partial(X, Y)=1$. Then the following (i), (ii) hold.

(i) $\left|D_{i-1}^{i}\right|=\left|D_{i}^{i-1}\right|=c_{i} k_{i} / b_{0}(1 \leq i \leq d)$.

(ii) $D_{j}^{i}=\emptyset$ if $|i-j| \neq 1(0 \leq i, j \leq d)$.

Proof. (i) This follows from [5, Lemma 4.1(i)].

(ii) By the triangle inequality we find $D_{j}^{i}=\emptyset$ if $|i-j| \geq 2$. Since $D_{d}(q)$ is bipartite, we also have $D_{i}^{i}=\emptyset$.

Lemma 3.2. With reference to Notation 1.1 assume that $\partial(X, Y)=1$. Pick $u \in X \backslash Y$ and $v \in Y \backslash X$. Then $f(u, v) \neq 0$. In particular, $u$ and $v$ are not contained in a common isotropic subspace.

Proof. Suppose on the contrary that $f(u, v)=0$. Pick $\lambda, \mu \in G F(q)$ and $w \in X \cap Y$. Consider $\lambda u+w+\mu v \in\langle X, Y\rangle$. By (1.2) and (1.4) we have

$Q(\lambda u+w+\mu v)=Q(\lambda u+w)+Q(\mu v)+f(\lambda u+w, \mu v)=\lambda \mu f(u, v)+\mu f(w, v)=0$.

This shows that $\langle X, Y\rangle$ is an isotropic subspace of dimension $d+1$, a contradiction.

Theorem 3.3. With reference to Notation 1.1 assume that $\partial(X, Y)=1$. Then the following (i), (ii) hold for $1 \leq i \leq d$.

(i) For every $Z, Z^{\prime} \in D_{i}^{i-1}$ there exists $\sigma \in G_{X} \cap G_{Y}$ which maps $Z$ to $Z^{\prime}$.

(ii) For every $Z, Z^{\prime} \in D_{i-1}^{i}$ there exists $\sigma \in G_{X} \cap G_{Y}$ which maps $Z$ to $Z^{\prime}$.

Proof. (i) If $i=1$ then the result is clear. Assume now that $i \geq 2$. Since $\operatorname{dim}(X \cap Z)=$ $d-i+1$ and $\operatorname{dim}(Y \cap Z)=d-i$, it follows from Lemma 3.2 that $X \cap Y \cap Z=Y \cap Z$ with $\operatorname{dim}(X \cap Y \cap Z)=d-i$, and $X \cap Z=\langle X \cap Y \cap Z, u\rangle$ for some $u \in X \backslash Y$. Pick $w \in Y \backslash X$. Let $v_{1}, \ldots, v_{d-1}$ be a basis of $X \cap Y$, such that $v_{i}, \ldots, v_{d-1}$ is a basis of $X \cap Y \cap Z$. Let $z_{1}, \ldots, z_{i-1} \in Z$ be such that $u, v_{i}, \ldots, v_{d-1}, z_{1}, \ldots, z_{i-1}$ is a basis of $Z$. Note that $u, v_{1}, \ldots, v_{d-1}$ is a basis of $X$ and that $w, v_{1}, \ldots, v_{d-1}$ is a basis of $Y$.

Similarly as above, let $u^{\prime} \in X \backslash Y$ be such that $X \cap Z^{\prime}=\left\langle X \cap Y \cap Z^{\prime}, u^{\prime}\right\rangle$. Let $v_{1}^{\prime}, \ldots, v_{d-1}^{\prime}$ be a basis of $X \cap Y$, such that $v_{i}^{\prime}, \ldots, v_{d-1}^{\prime}$ is a basis for $X \cap Y \cap Z^{\prime}$. Let $z_{1}^{\prime}, \ldots, z_{i-1}^{\prime} \in Z^{\prime}$ be such that $u^{\prime}, v_{i}^{\prime}, \ldots, v_{d-1}^{\prime}, z_{1}^{\prime}, \ldots, z_{i-1}^{\prime}$ is a basis for $Z^{\prime}$. Observe that $u^{\prime}, v_{1}^{\prime}, \ldots, v_{d-1}^{\prime}$ is a basis for $X$ and that $w, v_{1}^{\prime}, \ldots, v_{d-1}^{\prime}$ is a basis for $Y$.

Applying Lemma 2.3 (with $U=X=\left\langle u, v_{1}, \ldots, v_{d-1}\right\rangle, W=Z=\left\langle u, v_{i}, \ldots\right.$, $\left.v_{d-1}, z_{1}, \ldots, z_{i-1}\right\rangle, U_{1}=X=\left\langle u^{\prime}, v_{1}^{\prime}, \ldots, v_{d-1}^{\prime}\right\rangle$ and $W_{1}=Z^{\prime}=\left\langle u^{\prime}, v_{i}^{\prime}, \ldots, v_{d-1}^{\prime}\right.$, 
$\left.\left.z_{1}^{\prime}, \ldots, z_{i-1}^{\prime}\right\rangle\right)$ we find that there exists an isometry $\sigma$ such that $\sigma(u)=u^{\prime}, \sigma\left(v_{j}\right)=v_{j}^{\prime}(1 \leq$ $j \leq d-1)$, and $\sigma\left(z_{j}\right) \in\left\langle z_{1}^{\prime}, \ldots, z_{i-1}^{\prime}\right\rangle(1 \leq j \leq i-1)$. Clearly, $\sigma$ preserves $X$ (and thus also $X \cap Y$ ), and maps $Z$ to $Z^{\prime}$. To finish the proof we have to show that $\sigma$ preserves $Y$. Observe that $X \cap Y$ is a $(d-1)$-dimensional isotropic subspace of $V$. By Lemma 2.4, the only two maximal isotropic subspaces containing $X \cap Y$ are $X$ and $Y$. Since $X$ and $X \cap Y$ are both preserved by $\sigma$, also $Y$ is preserved by $\sigma$.

(ii) Similar as (i) above.

Proposition 3.4. With reference to Notation 1.1 assume that $\partial(X, Y)=1$. Then the following (i), (ii) hold.

(i) The set $D_{i}^{i-1}(1 \leq i \leq d)$ is an orbit of $G_{X} \cap G_{Y}$.

(ii) The set $D_{i-1}^{i}(1 \leq i \leq d)$ is an orbit of $G_{X} \cap G_{Y}$.

Proof. It is clear that two vertices from different sets from (i) and (ii) above could not be in the same orbit of $G_{X} \cap G_{Y}$. The result now follows from Theorem 3.3.

\section{The case $\partial(X, Y)=2$}

With reference to Notation 1.1, in this section we describe the orbits of $G_{X} \cap G_{Y}$ when $\partial(X, Y)=2$. We first determine the size of the sets $D_{j}^{i}(0 \leq i, j \leq d)$. The proposition below follows from [5, Lemma 4.1(ii)-(iv)].

Proposition 4.1. With reference to Notation 1.1 assume that $\partial(X, Y)=2$. Then the following (i)-(iv) hold.

(i) $\left|D_{i-2}^{i}\right|=\left|D_{i}^{i-2}\right|=k_{i} c_{i-1} c_{i} /\left(b_{0} b_{1}\right) \quad(2 \leq i \leq d)$;

(ii) $\left|D_{0}^{0}\right|=0$ and $\left|D_{i}^{i}\right|=k_{i}\left(c_{i}\left(b_{i-1}-1\right)+b_{i}\left(c_{i+1}-1\right)\right) /\left(b_{0} b_{1}\right) \quad(1 \leq i \leq d-1)$;

(iii) $\left|D_{d}^{d}\right|=k_{d}\left(b_{d-1}-1\right) / b_{1}$;

(iv) $\left|D_{j}^{i}\right|=0$ if $|i-j| \notin\{0,2\} \quad(0 \leq i, j \leq d)$.

Lemma 4.2. With reference to Notation 1.1 assume that $\partial(X, Y)=2$. Then the following (i), (ii) hold.

(i) Let $u_{1}, u_{2} \in X \backslash Y$ be linearly independent, and let $w \in Y \backslash X$. Then $u_{1}, u_{2}$ and $w$ are not contained in a common isotropic subspace of $V$.

(ii) Let $w_{1}, w_{2} \in Y \backslash X$ be linearly independent, and let $u \in X \backslash Y$. Then $w_{1}, w_{2}$ and $u$ are not contained in a common isotropic subspace of $V$.

Proof. (i) Suppose on contrary that $u_{1}, u_{2}$ and $w$ are contained in a common isotropic subspace. Pick $\lambda_{1}, \lambda_{2}, \mu \in G F(q)$ and $v \in X \cap Y$. Consider $\lambda_{1} u_{1}+\lambda_{2} u_{2}+v+\mu w \in$ $\langle X, w\rangle$. By (1.2) and (1.4) we have

$$
\begin{gathered}
Q\left(\lambda_{1} u_{1}+\lambda_{2} v_{2}+v+\mu w\right)=Q\left(\lambda_{1} u_{1}+\lambda_{2} u_{2}+v\right)+Q(\mu w)+f\left(\lambda_{1} u_{1}+\lambda_{2} u_{2}+v, \mu w\right)= \\
\lambda_{1} \mu f\left(u_{1}, w\right)+\lambda_{2} \mu f\left(u_{2}, w\right)+\mu f(v, w)=0 .
\end{gathered}
$$

Therefore, $\langle X, w\rangle$ is an isotropic subspace of dimension $d+1$, a contradiction.

(ii) Similar as (i) above. 
Theorem 4.3. With reference to Notation 1.1 assume that $\partial(X, Y)=2$. Then the following (i), (ii) hold for $2 \leq i \leq d$.

(i) For every $Z, Z^{\prime} \in D_{i}^{i-2}$ there exists $\sigma \in G_{X} \cap G_{Y}$ which maps $Z$ to $Z^{\prime}$.

(ii) For every $Z, Z^{\prime} \in D_{i-2}^{i}$ there exists $\sigma \in G_{X} \cap G_{Y}$ which maps $Z$ to $Z^{\prime}$.

Proof. (i) Note that the result is clear if $i=2$. Namely, for $i=2$ we have $Z=Z^{\prime}=X$. Assume now $i \geq 3$. By Lemma 4.2, there exists a basis $v_{1}, \ldots v_{d-2}$ of $X \cap Y$, vectors $u_{1}, u_{2} \in X$, vectors $w_{1}, w_{2} \in Y$, and vectors $z_{1}, \ldots z_{i-2} \in Z$, such that $v_{i-1}, \ldots, v_{d-2}$ is a basis of $X \cap Y \cap Z, u_{1}, u_{2}, v_{1}, \ldots v_{d-2}$ is a basis of $X, w_{1}, w_{2}, v_{1}, \ldots v_{d-2}$ is a basis of $Y$, and $u_{1}, u_{2}, v_{i-1}, \ldots v_{d-2}, z_{1}, \ldots, z_{i-2}$ is a basis of $Z$. Without loss of generality we can assume that $f\left(u_{1}, w_{1}\right)=0$ (otherwise we replace $w_{1}$ by $w_{1}+\lambda w_{2}$ for an appropriate $\lambda \in G F(q)$ ). This implies that $\left\langle X \cap Y, u_{1}, w_{1}\right\rangle$ is maximal isotropic subspace.

Similarly, there exists a basis $v_{1}^{\prime}, \ldots v_{d-2}^{\prime}$ of $X \cap Y$, vectors $u_{1}^{\prime}, u_{2}^{\prime} \in X$ and vectors $z_{1}^{\prime}, \ldots z_{i-2}^{\prime} \in Z^{\prime}$, such that $v_{i-1}^{\prime}, \ldots, v_{d-2}^{\prime}$ is a basis of $X \cap Y \cap Z^{\prime}, u_{1}^{\prime}, u_{2}^{\prime}, v_{1}^{\prime}, \ldots v_{d-2}^{\prime}$ is a basis of $X, w_{1}, w_{2}, v_{1}^{\prime}, \ldots v_{d-2}^{\prime}$ is a basis of $Y$, and $u_{1}^{\prime}, u_{2}^{\prime}, v_{i-1}^{\prime}, \ldots v_{d-2}^{\prime}, z_{1}^{\prime}, \ldots, z_{i-2}^{\prime}$ is a basis of $Z^{\prime}$. Without loss of generality we can assume that $f\left(u_{1}^{\prime}, w_{1}\right)=0$ (otherwise we replace $u_{1}^{\prime}$ by $u_{1}^{\prime}+\lambda u_{2}^{\prime}$ for an appropriate $\lambda \in G F(q)$ ). This implies that $\left\langle X \cap Y, u_{1}^{\prime}, w_{1}\right\rangle$ is maximal isotropic subspace.

Applying Lemma 2.3 (with $U=\left\langle u_{1}, w_{1}, v_{1}, \ldots, v_{d-2}\right\rangle, U_{1}=\left\langle u_{1}^{\prime}, w_{1}, v_{1}^{\prime}, \ldots, v_{d-2}^{\prime}\right\rangle$, $W=Z$ and $W_{1}=Z^{\prime}$ ) we find that there exists an isometry $\sigma$ of $V$, such that $\sigma\left(u_{1}\right)=u_{1}^{\prime}$, $\sigma\left(w_{1}\right)=w_{1}, \sigma\left(v_{j}\right)=v_{j}^{\prime}$ for $1 \leq j \leq d-2$, and $\sigma\left(u_{2}\right), \sigma\left(z_{j}\right) \in\left\langle u_{2}^{\prime}, z_{1}^{\prime}, \ldots, z_{i-2}^{\prime}\right\rangle(1 \leq$ $j \leq i-2)$. Clearly, $\sigma$ maps $Z$ to $Z^{\prime}$. It remains to show that $\sigma$ preserves $X$ and $Y$. Consider the subspace $W=\left\langle X \cap Y, u_{1}\right\rangle$. Note that $W$ is a $(d-1)$-dimensional isotropic subspace of $V$. By Lemma 2.4, the only two maximal isotropic subspaces containing $W$ are $X$ and $\left\langle W, w_{1}\right\rangle$. Isometry $\sigma$ maps $W$ to $W^{\prime}=\left\langle X \cap Y, u_{1}^{\prime}\right\rangle$. By Lemma 2.4, the only two maximal isotropic subspaces containing $W^{\prime}$ are $X$ and $\left\langle W^{\prime}, w_{1}\right\rangle$. Since $\sigma$ maps $\left\langle W, w_{1}\right\rangle$ to $\left\langle W^{\prime}, w_{1}\right\rangle$, it must map $X$ to $X$. Similarly we show that $\sigma$ maps $Y$ to $Y$. It follows that $\sigma \in G_{X} \cap G_{Y}$, completing the proof of (i).

(ii) Similarly as (i) above.

Let us now consider the sets $D_{i}^{i}(1 \leq i \leq d)$. Pick $Z \in D_{i}^{i}$. By Lemma 4.2, two essentially different situations can occur: either $\operatorname{dim}(X \cap Y \cap Z)=d-i$ (and therefore $X \cap Z=Y \cap Z=X \cap Y \cap Z)$, or $\operatorname{dim}(X \cap Y \cap Z)=d-i-1$ (and therefore $X \cap Z \neq Y \cap Z)$.

Definition 4.4. With reference to Notation 1.1 assume that $\partial(X, Y)=2$. Let $Z \in D_{i}^{i}(1 \leq$ $i \leq d)$. We say $Z$ is of positive (negative, respectively) type, whenever $\operatorname{dim}(X \cap Y \cap Z)=$ $d-i(\operatorname{dim}(X \cap Y \cap Z)=d-i-1$, respectively).

Observe that all vertices of $D_{1}^{1}$ are of negative type, and that all vertices of $D_{d}^{d}$ are of positive type. Moreover, every $D_{i}^{i}(2 \leq i \leq d-1)$ is a disjoint union of the set of vertices of $D_{i}^{i}$ of positive type, and the set of vertices of $D_{i}^{i}$ of negative type.

Remark 4.5. In [6], the definition of the vertices of positive (negative, respectively) type is different from Definition 4.4 above. Namely, $Z \in D_{i}^{i}$ is defined to be of positive type, whenever all vertices in $D_{1}^{1}$ are at distance $i-1$ from $Z$. On the other hand, $Z$ is defined to be of negative type, if there exists a vertex in $D_{1}^{1}$ which is at distance $i-1$ from $Z$, and all other vertices in $D_{1}^{1}$ are at distance $i+1$ from $Z$. However, these definitions are equivalent. 
If $\operatorname{dim}(X \cap Y \cap Z)=d-i$, then $Z$ is at distance at most $i$ from every vertex in $D_{1}^{1}$. By the triangle inequality and since $D_{d}(q)$ is bipartite, $Z$ is at distance $i-1$ from every vertex of $D_{1}^{1}$. On the other hand, if $\operatorname{dim}(X \cap Y \cap Z)=d-i-1$, then pick $u \in(X \cap Z) \backslash Y$ and $v \in(Y \cap Z) \backslash X$. Then $W=\langle X \cap Y, u, v\rangle$ is a vertex of $D_{d}(q)$, which belongs to $D_{1}^{1}$ and is at distance $i-1$ from $Z$. Furthermore, all other vertices in $D_{1}^{1}$ are at distance $i+1$ from $Z$.

Lemma 4.6. ([6, Theorem 5.3(iv),(v) and Proposition 6.3]) With reference to Notation 1.1 assume that $\partial(X, Y)=2$. Then the following (i), (ii) hold for $2 \leq i \leq d-1$.

(i) $\mid\left\{z \in D_{i}^{i} \mid z\right.$ is of positive type $\} \mid=k_{i}(q-1) c_{i} c_{i-1} /\left(b_{0} b_{1}\right)$;

(ii) $\mid\left\{z \in D_{i}^{i} \mid z\right.$ is of negative type $\} \mid=k_{i} b_{i} c_{i} c_{2} /\left(b_{0} b_{1}\right)$.

Theorem 4.7. With reference to Notation 1.1 assume that $\partial(X, Y)=2$. Let $Z, Z^{\prime} \in$ $D_{i}^{i}(1 \leq i \leq d-1)$ and assume $Z, Z^{\prime}$ are of negative type. Then there exists $\sigma \in G_{X} \cap G_{Y}$ which maps $Z$ to $Z^{\prime}$.

Proof. Let $v_{1}, \ldots, v_{d-2}$ be a basis of $X \cap Y$ such that $v_{i}, \ldots, v_{d-2}$ is a basis of $X \cap$ $Y \cap Z$. Let $u_{1} \in X$ and $w_{1} \in Y$ be such that $u_{1}, v_{i}, \ldots, v_{d-2}$ is a basis of $X \cap Z$ and such that $w_{1}, v_{i}, \ldots, v_{d-2}$ is a basis of $Y \cap Z$. Let $u_{2} \in X$ and $w_{2} \in Y$ be such that $u_{1}, u_{2}, v_{1}, \ldots, v_{d-2}$ is a basis of $X$ and such that $w_{1}, w_{2}, v_{1}, \ldots, v_{d-2}$ is a basis of $Y$. Finally, let $z_{1}, \ldots, z_{i-1} \in Z$ be such that $u_{1}, w_{1}, z_{1}, \ldots, z_{i-1}, v_{i}, \ldots, v_{d-2}$ is a basis of $Z$.

Similarly, let $v_{1}^{\prime}, \ldots, v_{d-2}^{\prime}$ be a basis of $X \cap Y$ such that $v_{i}^{\prime}, \ldots, v_{d-2}^{\prime}$ is a basis of $X \cap Y \cap Z^{\prime}$. Let $u_{1}^{\prime} \in X$ and $w_{1}^{\prime} \in Y$ be such that $u_{1}^{\prime}, v_{i}^{\prime}, \ldots, v_{d-2}^{\prime}$ is a basis of $X \cap Z^{\prime}$ and such that $w_{1}^{\prime}, v_{i}^{\prime}, \ldots, v_{d-2}^{\prime}$ is a basis of $Y \cap Z^{\prime}$. Let $u_{2}^{\prime} \in X$ and $w_{2}^{\prime} \in Y$ be such that $u_{1}^{\prime}, u_{2}^{\prime}, v_{1}^{\prime}, \ldots, v_{d-2}^{\prime}$ is a basis of $X$ and such that $w_{1}^{\prime}, w_{2}^{\prime}, v_{1}^{\prime}, \ldots, v_{d-2}^{\prime}$ is a basis of $Y$. Finally, let $z_{1}^{\prime}, \ldots, z_{i-1}^{\prime} \in Z^{\prime}$ be such that $u_{1}^{\prime}, w_{1}^{\prime}, z_{1}^{\prime}, \ldots, z_{i-1}^{\prime}, v_{i}^{\prime}, \ldots, v_{d-2}^{\prime}$ is a basis of $Z^{\prime}$.

Applying Lemma 2.3 (with $U=\left\langle u_{1}, w_{1}, v_{1}, \ldots, v_{d-2}\right\rangle, U_{1}=\left\langle u_{1}^{\prime}, w_{1}^{\prime}, v_{1}^{\prime}, \ldots, v_{d-2}^{\prime}\right\rangle$, $W=Z$ and $W_{1}=Z^{\prime}$ ) we find that there exists an isometry $\sigma$ such that $\sigma\left(u_{1}\right)=u_{1}^{\prime}$, $\sigma\left(w_{1}\right)=w_{1}^{\prime}, \sigma\left(v_{j}\right)=v_{j}^{\prime}(1 \leq j \leq d-2)$, and $\sigma\left(z_{j}\right) \in\left\langle z_{1}^{\prime}, \ldots, z_{i-1}^{\prime}\right\rangle$ for $1 \leq j \leq i-1$. Clearly, $\sigma$ maps $Z$ to $Z^{\prime}$. It remains to show that $\sigma$ preserves $X$ and $Y$. Note that $W=$ $\left\langle X \cap Y, u_{1}\right\rangle$ is a $(d-1)$-dimensional isotropic subspace of $V$. By Lemma 2.4, the only two maximal isotropic subspaces containing $W$ are $X$ and $\left\langle W, w_{1}\right\rangle$. Note that $\sigma$ maps $W$ to $W^{\prime}=\left\langle X \cap Y, u_{1}^{\prime}\right\rangle$, which is a $(d-1)$-dimensional isotropic subspace of $V$. The only two maximal isotropic subspaces containing $W^{\prime}$ are $X$ and $\left\langle W^{\prime}, w_{1}^{\prime}\right\rangle$. Since $\sigma$ maps $\left\langle W, w_{1}\right\rangle$ to $\left\langle W^{\prime}, w_{1}^{\prime}\right\rangle$, it must map $X$ to $X$. Similarly we show that $\sigma$ maps $Y$ to $Y$. Therefore $\sigma \in G_{X} \cap G_{Y}$ and the proof is completed.

Theorem 4.8. With reference to Notation 1.1 assume that $\partial(X, Y)=2$. Let $Z, Z^{\prime} \in$ $D_{i}^{i}(2 \leq i \leq d)$ and assume $Z, Z^{\prime}$ are of positive type. Then there exist $\sigma \in G_{X} \cap G_{Y}$ which maps $Z$ to $Z^{\prime}$.

Proof. Let $v_{1}, \ldots, v_{d-2}$ be a basis of $X \cap Y$ such that $v_{i-1}, \ldots, v_{d-2}$ is a basis of $X \cap$ $Y \cap Z$. Let $u_{1}, u_{2} \in X$ and $w_{1}, w_{2} \in Y$ be such that $u_{1}, u_{2}, v_{1}, \ldots, v_{d-2}$ is a basis of $X$ and $w_{1}, w_{2}, v_{1}, \ldots, v_{d-2}$ is a basis of $Y$. Without loss of generality we can assume that $f\left(u_{1}, w_{1}\right)=0$ (otherwise we replace $w_{1}$ by $w_{1}+\lambda w_{2}$ for an appropriate $\lambda \in G F(q)$ ). Note that $\left\langle X \cap Y, u_{1}, w_{1}\right\rangle \in D_{1}^{1}$. Since $Z$ is of positive type we have $\operatorname{dim}\left(\left\langle X \cap Y, u_{1}, w_{1}\right\rangle \cap Z\right)=$ $d-i+1$. Therefore, there exist $\alpha, \beta \in G F(q)$ and $v \in X \cap Y$ such that $\left\langle X \cap Y, u_{1}, w_{1}\right\rangle \cap$ 
$Z=\left\langle\alpha u_{1}+\beta w_{1}+v, v_{i-1}, \ldots, v_{d-2}\right\rangle$. Since $\operatorname{dim}(X \cap Z)=\operatorname{dim}(Y \cap Z)=d-i$, we have $\alpha \neq 0$ and $\beta \neq 0$. Without loss of generality we can therefore assume that $\langle X \cap$ $\left.Y, u_{1}, w_{1}\right\rangle \cap Z=\left\langle u_{1}+w_{1}, v_{i-1}, \ldots, v_{d-2}\right\rangle$ (otherwise we replace $u_{1}$ by $\alpha u_{1}+v$ and $w_{1}$ by $\left.\beta w_{1}\right)$. Finally, let $z_{1}, \ldots, z_{i-1} \in Z$ be such that $z_{1}, \ldots, z_{i-1}, u_{1}+w_{1}, v_{i-1}, \ldots, v_{d-2}$ is a basis of $Z$.

Similarly, Let $v_{1}^{\prime}, \ldots, v_{d-2}^{\prime}$ be a basis of $X \cap Y$ such that $v_{i-1}^{\prime}, \ldots, v_{d-2}^{\prime}$ is a basis of $X \cap Y \cap Z^{\prime}$. Let $u_{1}^{\prime}, u_{2}^{\prime} \in X$ and $w_{1}^{\prime}, w_{2}^{\prime} \in Y$ be such that $u_{1}^{\prime}, u_{2}^{\prime}, v_{1}^{\prime}, \ldots, v_{d-2}^{\prime}$ is a basis of $X$ and $w_{1}^{\prime}, w_{2}^{\prime}, v_{1}^{\prime}, \ldots, v_{d-2}^{\prime}$ is a basis of $Y$. Without loss of generality we can assume that $f\left(u_{1}^{\prime}, w_{1}^{\prime}\right)=0$ and that $\left\langle X \cap Y, u_{1}^{\prime}, w_{1}^{\prime}\right\rangle \cap Z^{\prime}=\left\langle u_{1}^{\prime}+w_{1}^{\prime}, v_{i-1}^{\prime}, \ldots, v_{d-2}^{\prime}\right\rangle$. Let $z_{1}^{\prime}, \ldots, z_{i-1}^{\prime} \in Z^{\prime}$ be such that $z_{1}^{\prime}, \ldots, z_{i-1}^{\prime}, u_{1}^{\prime}+w_{1}^{\prime}, v_{i-1}^{\prime}, \ldots, v_{d-2}^{\prime}$ is a basis of $Z^{\prime}$.

Applying Lemma 2.3 (with $U=\left\langle u_{1}, u_{1}+w_{1}, v_{1}, \ldots, v_{d-2}\right\rangle, W=Z, U_{1}=\left\langle u_{1}^{\prime}, u_{1}^{\prime}+\right.$ $\left.w_{1}^{\prime}, v_{1}^{\prime}, \ldots, v_{d-2}^{\prime}\right\rangle$ and $\left.W_{1}=Z^{\prime}\right)$ we find that there exists an isometry $\sigma$ of $V$ such that $\sigma\left(u_{1}\right)=u_{1}^{\prime}, \sigma\left(u_{1}+w_{1}\right)=u_{1}^{\prime}+w_{1}^{\prime}$ (and therefore also $\left.\sigma\left(w_{1}\right)=w_{1}^{\prime}\right), \sigma\left(v_{j}\right)=v_{j}^{\prime}(1 \leq$ $j \leq d-2)$, and $\sigma\left(z_{j}\right) \in\left\langle z_{1}^{\prime}, \ldots, z_{i-1}^{\prime}\right\rangle$ for $1 \leq j \leq i-1$. Clearly, $\sigma$ maps $Z$ to $Z^{\prime}$. It remains to show $\sigma$ preserves $X$ and $Y$.

Note that $W=\left\langle X \cap Y, u_{1}\right\rangle$ is a $(d-1)$-dimensional isotropic subspace of $V$. By Lemma 2.4, the only two maximal isotropic subspaces containing $W$ are $X$ and $\left\langle W, w_{1}\right\rangle$. Note that $\sigma$ maps $W$ to $W^{\prime}=\left\langle X \cap Y, u_{1}^{\prime}\right\rangle$, which is a $(d-1)$-dimensional isotropic subspace of $V$. The only two maximal isotropic subspaces containing $W^{\prime}$ are $X$ and $\left\langle W^{\prime}, w_{1}^{\prime}\right\rangle$. Since $\sigma$ maps $\left\langle W, w_{1}\right\rangle$ to $\left\langle W^{\prime}, w_{1}^{\prime}\right\rangle$, it must map $X$ to $X$. Similarly we show that $\sigma$ maps $Y$ to $Y$. Therefore $\sigma \in G_{X} \cap G_{Y}$ and the proof is complete.

Proposition 4.9. With reference to Notation 1.1 assume that $\partial(X, Y)=2$. Then the following (i)-(iii) hold.

(i) Each of $D_{1}^{1}, D_{d}^{d}$ is an orbit of $G_{X} \cap G_{Y}$.

(ii) For $2 \leq i \leq d$ the sets $D_{i-2}^{i}$ and $D_{i}^{i-2}$ are orbits of $G_{X} \cap G_{Y}$.

(iii) For $2 \leq i \leq d-1$ the set of vertices in $D_{i}^{i}$ that are of positive type (resp. negative type) is an orbit of $G_{X} \cap G_{Y}$.

Proof. Observe that two vertices of $D_{d}(q)$, which are contained in distinct sets listed in (i), (ii) and (iii) above, cannot belong to the same orbit of $G_{X} \cap G_{Y}$. The result now follows from Theorems 4.3, 4.7 and 4.8.

\section{Acknowledgement}

The author would like to thank to Klavdija Kutnar and Pablo Spiga for many valuable suggestions.

\section{References}

[1] E. Artin, Geometric Algebra, Interscience, New York, 1957.

[2] A. E. Brouwer, A. M. Cohen and A. Neumaier, Distance-Regular Graphs, Springer-Verlag, Berlin, Heidelberg, 1989.

[3] B. Curtin, Bipartite distance-regular graphs I, Graphs Combin. 15 (1999), 143-157.

[4] B. Curtin, Bipartite distance-regular graphs II, Graphs Combin. 15 (1999), 377-391.

[5] Š. Miklavič, On bipartite $Q$-polynomial distance-regular graphs, European J. Combin. 28 (2007), 94-110. 
[6] $\check{S}$. Miklavič, On bipartite distance-regular graphs with intersection numbers $c_{i}=\left(q^{i}-1\right) /(q-$ 1), submitted to Graphs Combin.

[7] P. Terwilliger, The subconstituent algebra of an association scheme I, J. Algebraic Combin. 1 (1992), 363-388.

[8] P. Terwilliger, The subconstituent algebra of an association scheme II, J. Algebraic Combin. 2 (1993), 73-103.

[9] P. Terwilliger, The subconstituent algebra of an association scheme III, J. Algebraic Combin. 2 (1993), 177-210.

[10] V. A. Vasilev and V. D. Mazurov, Minimal permutation representations of finite simple orthogonal groups, Algebra Logic 33 (1994), 337-350. 